(REVIEW ARTICLE)

\title{
Practical guide and some recommendations for the diagnosis and management of Attention deficit hyperactivity disorder (ADHD)
}

\author{
Petlovanyi Petro * and Tsarkov Anatolii \\ Department of Psychiatry, School of Medicine, University of Zambia (UNZA).
}

Publication history: Received on 18 June 2020; revised on 25 June 2020; accepted on 27 June 2020

Article DOI: https://doi.org/10.30574/wjarr.2020.6.3.0209

\begin{abstract}
Attention deficit hyperactivity disorder (ADHD) is described as the most common neurobehavioral condition of childhood. Symptoms include difficulty concentrating, hyperactivity and poorly controlled impulsivity. ADHD always manifests in childhood, children with the syndrome rush around the room, switch their attention from one to the other, cannot calm down and take lessons or reading. ADHD may persist into adulthood causing disruptions to both professional and personal life. The pharmacotherapy of ADHD is cost-effective, but treatment should be individual. Main outlines of clinical practice guide on management of ADHD of Academy of Medicine and Ministry of Health of Singapore are discussed in this article. Some focus on diagnostic process and associated with ADHD problems is emphasized. It can be invaluable example for the specialists engaged in the treatment of the children with this psychiatric disorder.
\end{abstract}

Keywords: ADHD; Child psychiatry; Methylphenidate; Atomoxetine; Omega-3; Bipolar disorder

\section{Introduction}

Attention deficit hyperactivity disorder (ADHD) - is the most common disorder that occurs in pediatric psychiatric practice. ADHD is characterized by motor hyperactivity, disorder of attention and impulsivity and can lead to pronounced impairments of all major functioning areas of the child. According to available data, the prevalence of ADHD in the population of preschool children is $1.5-9 \%$ and among schoolchildren $-6-19 \%$, while in $50 \%$ cases the symptoms of this disorder can occur in adulthood [1-4]. While ADHD manifestations have a negative impact on all life activities both for the affected person and society as a whole. Herewith, it is known that the timely delivery of health care for such patients can be effective. Key provisions of clinical practice guide on management of ADHD of Academy of Medicine and Ministry of Health of Singapore are presented in this article. Important aspects of associated with ADHD problems and diagnostic process are discussed.

\section{Definition and diagnostic classification}

ADHD diagnosis should be established by clinical judgment that includes a mandatory interview with the parents or caregivers of the child (grade D recommendation, level of evidence 2++) [2, 5-6]. In addition, in the process of ADHD evaluating it is important to obtain information from people who interact with the patient in other conditions, for example, teachers at school (grade B recommendation, level of evidence $2++$ ).

Before making a conclusion that a child suffers from ADHD, it is necessary to perform a thorough diagnostic evaluation to exclude other psychiatric medical disorders, symptoms, which can be mistaken for manifestations resembling ADHD symptoms (grade B recommendation, level of evidence $2++$ ). It is important to note that in preschool children with destructive behaviour, manifestation of ADHD is possible (grade B recommendation, level of evidence 2++). In case of patients with an established diagnosis of ADHD, a clinician should conduct an assessment of comorbid conditions (grade $\mathrm{C}$ recommendation, level of evidence 2+) [2,5-8]. If there is a suspicion that a child has learning disorders, it is necessary

\footnotetext{
* Corresponding author: Petlovanyi Petro
} 
to perform the appropriate language assessment using physiological and educational programs (grade $\mathrm{C}$ recommendation, level of evidence $2+$ ).

For patients with the ADHD diagnosis, in the absence of other medical evidences, it is not necessary to evaluate thyroid function test as well as carry out neuroimaging examination (grade A recommendation, level of evidence 1+). In addition, in clinical practice electroencephalography (EEG) is not recommended as ADHD diagnostic tool (grade A recommendation, level of evidence $1+$ ).

Dental health in children with ADHD may be compromised [9-10]. Children with ADHD may present with behavioral management problems in the dental setting due to increased anxiety and fear. These children also have a higher risk of suffering from traumatic dental and orofacial injuries.

Studies have been conducted to examine the relationship between the presence of ADHD and alcohol abuse, drug use and smoking [12]. ADHD is 5 to 10 times more common among adult alcoholics than it is in people without the condition. Among adults being treated for alcohol and substance abuse, the rate of ADHD is about 25\% [12]. Research of other addictive behaviors suggests an association between ADHD and gambling addiction [13]. Pathological Gambling and ADHD share a common feature of impulsivity [13-14].

The levels of evidence and grades of recommendation are shown in the Table 1.

Table 1 Levels of evidence and recommendation grades

\begin{tabular}{|c|c|}
\hline \multicolumn{2}{|c|}{ Levels of evidence } \\
\hline Level & Type of evidence \\
\hline $1++$ & $\begin{array}{l}\text { High quality meta-analyses, systematic reviews of randomized controlled trials (RCTs), or } \\
\text { RCTs with a very low risk of bias }\end{array}$ \\
\hline $1+$ & Well conducted meta-analyses, systematic reviews of RCTs, or RCTs with a low risk of bias \\
\hline 1- & Meta-analyses, systematic reviews of RCTs, or RCTs with a high risk of bias \\
\hline $2++$ & $\begin{array}{l}\text { High quality systematic reviews of case control or cohort studies. High quality case control } \\
\text { or cohort studies with a very low risk of confounding or bias and a high probability that the } \\
\text { relationship is causal }\end{array}$ \\
\hline $2+$ & $\begin{array}{l}\text { Well conducted case control or cohort studies with a low risk of confounding or bias and a } \\
\text { moderate probability that the relationship is causal }\end{array}$ \\
\hline $2-$ & $\begin{array}{l}\text { Case control or cohort studies with a high risk of confounding or bias and a significant risk } \\
\text { that the relationship is not causal }\end{array}$ \\
\hline 3 & Non-analytic studies, e.g. case reports, case series \\
\hline 4 & Expert opinion \\
\hline
\end{tabular}

\section{Recommendation grades}

Grade Recommendation

A

B

C

D

GPP (good Recommended best practice based on the clinical experience of the guideline development practice group

At least one meta-analysis, systematic review of RCTs, or RCT rated as 1++ and directly applicable to the target population; or A body of evidence consisting principally of studies rated as $1+$, directly applicable to the target population, and demonstrating overall consistency of results

A body of evidence including studies rated as $2++$, directly applicable to the target population, and demonstrating overall consistency of results; or Extrapolated evidence from studies rated as $1++$ or $1+$

A body of evidence including studies rated as 2+, directly applicable to the target population and demonstrating overall consistency of results; or Extrapolated evidence from studies rated as $2++$

Evidence level 3 or 4; or Extrapolated evidence from studies rated as 2+

points) 


\section{Management of the patients with ADHD}

Specialists managing children with ADHD have to plan long-term strategies for management and rehabilitation and always discuss it with patients and their families, as well as to ensure the possibility of further assistance (grade D recommendation, level of evidence 4).

\subsection{Psychological, alternative and other additional interventions}

After diagnosing ADHD, clinicians should ensure improvement of the awareness of children, families and teachers about the disorder (grade B recommendation, level of evidence 1+). The physician should consider training the parents of patients with ADHD on behaviour management strategies or refer them to an appropriate specialist such as psychologist and occupational therapist (grade A recommendation, level of evidence 1++). Parents of preschool children with ADHD should be recommended to attend training for parents (grade B recommendation, level of evidence 1+). In addition, attending physician may consider referral of parents for public training programs, in particular, if the negative parenting practice is identified (degree of recommendation $\mathrm{B}$, level of evidence 1+). When conducting such training, it is recommended to apply the most appropriate strategy to control the behaviour with regard to their understanding and cultural background (level of recommendation D, level of evidence 3). Family therapy can also be considered, if there is a serious breach of family relationships

(GPP). Additionally, it is advisable to consider conducting of training (academic) interventions for children with ADHD and consultation with teachers who work closely with the child in the process of schooling (grade of recommendation $\mathrm{C}$, level of evidence 2+). It should be also explained to parents and teachers, that providing information about success and the child's behaviour in school can be useful (GPP).

In the management of patients with ADHD it is not recommended to conduct only social skills training (degree of recommendation $B$, level of evidence $1+$ ) or cognitive behavioural therapy (grade of recommendation $B$, level of evidence 1+), or biofeedback (degree of recommendation B, level of evidence 1+). In addition, it is not recommended to use only one recovering cognitive therapy in case of ADHD with significant cognitive disorders (grade of recommendation $A$, level of evidence $1+$ ).

A referral to an occupational therapist can be considered for children with sensory and motor disorders as supplementary treatment of ADHD (grade of recommendation D, level of evidence 3).

It was found that clear evidences for use of nutritional supplements and carbohydrates in cases of ADHD are not available. Parents and children should be encouraged to monitor the ingestion of products containing dietary supplements or large amounts of carbohydrates in order to monitor physical or behavioural manifestations (grade of recommendation $B$, level of evidence $1+$ ). In the management of patients with ADHD a restrictive diet is not recommended (grade of recommendation $C$, level of evidence 2++). Supplements of Omega-3 can be used as a complementary treatment of ADHD (grade e of recommendation B, level of evidence 1+) [2-3].

\subsection{Psychopharmacological treatment}

When making a decision to start drug therapy of ADHD, the use of methylphenidate should be considered in the first place (grade of recommendation A, level of evidence 1+). To treat symptoms of ADHD methylphenidate can be used for a long period of time, however, the effectiveness of the therapy should be regularly checked (grade of recommendation B, level of evidence 1+). In order to limit the development of adverse effects related to treatment, "drug holidays" can be considered. In the days free of drug, the symptoms of ADHD and their deterioration should be monitored (grade of recommendation $B$, level of evidence $1+$ ). In children administering methylphenidate, regular monitoring of height, weight and body mass index (grade of recommendation A, level of evidence 1++) is required. If there is a concern of impaired development, a continuation of pharmacotherapy should be considered and a decision should be made jointly with the parents. It is also necessary to study other probable medical causes that could determine it (grade of recommendation $\mathrm{D}$, level of evidence 4 ).

Prior to prescribing a therapy for ADHD, it is advisable to pay special attention to personal and family history of cardiovascular diseases. Children with previously identified cardiac problems should be referred for consultation to a cardiologist before beginning the therapy with methylphenidate or atomoxetine (grade of recommendation $\mathrm{C}$, level of evidence 2+). Some patients with ADHD may be at higher risk for stroke, heart attack, and sudden death, so any medication should be monitored for its effects on cardiovascular health [14-15]. Methylphenidate may create problems for older patients with cardiac or blood pressure issues. This medication can be prescribed for children and adolescents suffering from ADHD with the accompanying tic disorder, but the treatment should be stopped if any deterioration of 
tic is found (grade of recommendation A, level of evidence 1+) [2, 5-6]. Therapy with methylphenidate in the presence of comorbid destructive behavioural disorders should be considered (grade of recommendation B, level of evidence 1+). Furthermore, the treatment of ADHD with methylphenidate can be recommended for children and adolescents with autism spectrum disorder. In the process of management of such patients, special precautions and careful monitoring of side effects (grade of recommendation A, level of evidence 1+) should be taken. The treatment with methylphenidate should be started with low doses by slow titration according to therapy response, with necessary time correction of the drug administration for minimizing short-term side effects (grade of recommendation B, level of evidence 1+). It is recommended to consider the use of methylphenidate with slow release instead of methylphenidate with rapid release, if there are concerns on abuse of the drug. Such patients should be carefully monitored concerning drug administration (grade of recommendation $\mathrm{B}$, level of evidence $1+$ ).

Atomoxetine can be prescribed for the treatment of ADHD symptoms with high risk of methylphenidate abuse or related side effects (grade of recommendation A, level of evidence 1+). In the process of treatment with atomoxetine, the child should be periodically monitored concerning the indicators of development - growth, weight and body mass index every six months (grade of recommendation D, level of evidence 4) and mental state (suicidal ideas). If there are concerns on developmental delay, it is recommended to consider the need for further drug administration and make a decision jointly with the parents, as well as to evaluate other medical causes, probably, related to it (degree of recommendation $\mathrm{A}$, level of evidence $1++$ ). In addition, atomoxetine can be used as first-line treatment of ADHD in case of comorbidity of tic disorder (grade of recommendation $B$, level of evidence $1+$ ).

It is important to note that the use of combination of methylphenidate and atomoxetine for treating the symptoms of ADHD is not recommended (grade of recommendation C, level of evidence 2+). The use of methylphenidate or atomoxetine in preschool children should only be considered in case of ineffectiveness of psychosocial interventions. It is necessary to take careful precautions and monitor side effects when deciding about the continuation of drug administration (grade of recommendation A, level of evidence 1++). To improve the compliance with prescribed treatment for each patient with ADHD, both preferences of the children and their parents should be considered (grade of recommendation $\mathrm{A}$, level of evidence $1+$ ).

Diagnosis and treatment of comorbid conditions (depression, bipolar disorder, eating disorders, anxiety disorders and behavioral disorders) is an integral step in the comprehensive treatment of ADHD [2-3, 5-8, 16].

\section{Conclusion}

Currently, Zambia and other Sub-Saharan countries do not have permanent guidelines for the treatment of children and adolescents with ADHD. Singapore ADHD treatment protocol discussed in this article can be invaluable example for the specialists engaged in the treatment of the children with this psychiatric disorder. It could also serve as a strong example for the creation of the national guidelines for the ADHD treatment. Despite the fact that pharmacotherapy of ADHD is cost-effective, treatment should be individual. Possible presence of comorbid conditions should be taken into consideration before treatment of the disorder.

\section{Compliance with ethical standards}

\section{Acknowledgments}

Authors wish to acknowledge continuous help and support of Professor Trevor Kaile (Dean, School of Medicine, University of Zambia - UNZA), Doctor Elliot Kafumukache (Acting Dean, School of Medicine, University of Zambia UNZA), and Doctor Ravi Paul (Head of Department, Department of Psychiatry, University of Zambia - UNZA), who made writing this paper possible.

\section{Disclosure of conflict of interest}

There is no conflict of interest.

\section{References}

[1] Danielson ML, Visser SN, Chronis-Tuscano A and Du Paul GJ. (2018). A national description of treatment among U.S. children and adolescents with ADHD. Journal of Pediatrics, 192, 240-246.

[2] Attention Deficit Hyperactivity Disorder. AMS-MOH Clinical Practice Guidelines 1/2014, May 2014. 
[3] Tsarkov A and Petlovanyi P. (2017). Omega-3 Fatty Acids as an Alternative Treatment for Children with Attention Deficit Hyperactivity Disorder. Imperial Journal of Interdisciplinary Research (IJIR), 3, 1378-80.

[4] Sadock BJ, Sadock VA and Ruiz P. (2015). Synopsis of Psychiatry. 11th Ed., Wolters Kluwer, 1169-1181.

[5] Tsarkov A and Petlovanyi P. Bipolar disorder in child psychiatric practice. The African Digital Health Library (ADHL).

[6] Tsarkov A and Petlovanyi P. (2016). Bipolar Disorder in Child Psychiatric Practice: A Case Report. Medical Journal of Zambia, 43(1), 41-6.

[7] Tsarkov A and Petlovanyi P. Depressive Disorder in Child Psychiatric Practice: A. The Health Press, 9.

[8] Petlovanyi P and Tsarkov A. (2020). Child Schizophrenia: Theory and Practice. European Journal of Medical and Health Sciences, 2(1).

[9] Chandra P, Anandakrishna L and Ray P. (2009). Caries experience and oral hygiene status of children suffering from attention deficit hyperactivity disorder. J Clin Pediatr Dent, 34(1), 25-29.

[10] Phiri C, Tsarkov A, Petlovanyi P and Lingenda G. Factors Contributing To Oral Diseases and Treatment Needs amongst Mental Patients at Chainama Hills College Hospital, Lusaka, Zambia.

[11] Mariani JJ and Levin FR. (2007). Treatment strategies for co-occurring ADHD and substance use disorders. Am J Addict. 16 Suppl 1(Suppl 1), 45-56.

[12] Mak C, Tan KK and Guo S. (2018). ADHD Symptoms in Pathological and Problem Gamblers in Singapore. Int J Environ Res Public Health. 15(7), 1307.

[13] Tsarkov A and Petlovanyi P. Pathological Gambling: The Old Problem of the Modern World.

[14] Ueno KI, Togashi H, Matsumoto M, Ohashi S, Saito H and Yoshioka M. (2002). $\alpha 4 \beta 2$ nicotinic acetylcholine receptor activation ameliorates impairment of spontaneous alternation behavior in stroke-prone spontaneously hypertensive rats, an animal model of attention deficit hyperactivity disorder. Journal of Pharmacology and Experimental Therapeutics, 302(1), 95-100.

[15] Tsarkov A and Petlovanyi P. (2019). Neuropsychiatric Aspects of a Common Problem: Stroke. European Journal of Medical and Health Sciences, 1(3).

[16] Tsarkov A and Petlovanyi P. The Role of Lamotrigine in the Treatment of Bipolar Depression.

\section{How to cite this article}

Petlovanyi P and Tsarkov A. (2020). Practical guide and some recommendations for the diagnosis and management of Attention deficit hyperactivity disorder (ADHD). World Journal of Advanced Research and Reviews, 6(3), 257-261. 